\title{
A Calculus Approach to Minimum Energy Transmission Policies with Quality of Service Guarantees
}

\author{
Murtaza A. Zafer \\ Eytan Modiano \\ murtaza@mit.edu \\ modiano@mit.edu \\ Laboratory for Information and Decision Systems \\ Massachusetts Institute of Technology
}

\begin{abstract}
We consider a queueing system with controllable service rate; for example, a transmitter whose rate can be controlled by varying the transmission power. For such a system we obtain optimal data transmission policies that satisfy given quality of service $(\mathrm{QoS})$ constraints and also minimize the total transmission energy expenditure. First, we consider the deterministic case of known arrivals and present a formulation based on a calculus approach using arrival and minimum departure curves. The problem is posed as a continuous time optimization and an optimal solution is obtained for general arrival curves and QoS constraints. In the latter half of the paper, we consider a stochastic arrival process (Poisson process) and a single deadline constraint. The objective is to obtain a transmission policy that minimizes the expected energy expenditure. The problem is formulated as a stochastic optimal control problem and an explicit solution is obtained with some relaxation. Finally, simulation results comparing various policies are presented.
\end{abstract}

\section{INTRODUCTION}

Energy efficiency is of prime importance in many wireless systems such as sensor and ad-hoc networks. In general, transmission energy forms a significant proportion of the total energy expenditure and hence, one way to maximize battery lifetime is by reducing transmission energy. This can be achieved by employing low rate transmissions. It has been argued in [1], [7] that transmitting an amount of data at a low rate but over a longer duration has less energy cost as compared to a fast rate transmission over a shorter duration. Mathematically, this means that the power required to transmit at a certain rate is a convex function of the rate. Such a convex power-rate relationship is widely used in the literature [2], [3], [4], [7].

In addition to energy concerns, data arriving to a queue is bursty with varying QoS requirements. In principle, one can meet these QoS requirements by transmitting at higher rates subject to causality constraints imposed by the arrival process. This requires higher transmission power and an increased energy expenditure. On the contrary, a lower rate transmission may not always be able to meet the QoS constraints. Thus, by choosing the transmission rate correctly we can reduce

This work was supported by NSF ITR grant CCR-0325401, by DARPA/AFOSR through the University of Illinois grant no. F49620-02-10325 and by NASA Space Communication Project grant number NAG3-2835. the transmission energy expenditure and also satisfy the QoS constraints.

Most of the work on QoS has been related to average guarantees such as throughput, average delay and average packet drop [7], [8], [9]. These quantities are measured over an infinite horizon and do not directly translate into any QoS guarantees over finite time intervals. Some of the recent work that deals with strict constraints includes [1], [2], [3], [4]. Our goal in this work is guaranteeing QoS over a finite time interval with minimum energy expenditure.

Our main contributions in this work are as follows. We present a novel formulation based on the new concept of a minimum departure curve. We show that for a given arrival curve the QoS constraints can be translated into a minimum departure curve constraint. Such an approach helps in modelling a wide variety of QoS constraints. These ideas are explained in detail in Section II-A. Some of the early work on the notions of arrival and departure curves includes [5], [6]. Using a calculus approach we formulate an energy optimization problem in continuous time and obtain the optimal solution. The graphical visualization of the problem is very appealing and helps understand the optimal solution in a simple and intuitive way. In Section III-B, we present a few examples that can be modelled with this approach and also point out a heuristic online policy that can constructed without any future knowledge or statistical information of the arrival curve. While these are illustrative examples, many other applications can be easily modelled within our framework.

We, then, extend the formulation and introduce stochasticity in the arrival process. We consider a queue with some initial data, a Poisson arrival process and a single deadline by which the queue must be empty. The problem has motivations in a polling system [11] (eg. sensor network) where a central entity polls individual transmitters for finite durations during which the transmitter empties its buffer and any new arrivals. It can also be viewed as a system offering service with a convex cost over a finite duration by which the unfinished work must be zero. We model the problem using continuoustime stochastic optimal control theory as illustrated in the selfcontained presentation in Section IV. The framework we adopt demonstrates an approach different from discrete dynamic 

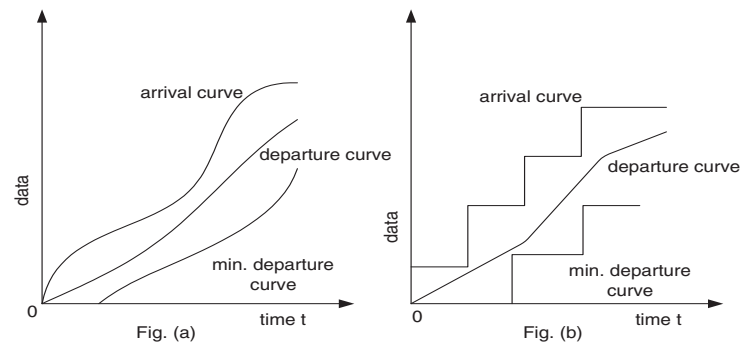

Fig. 1. Data flow model; (a) Continuous arrival model, (b) Packetized arrival model

programming and it leads to a simple and elegant solution for this otherwise difficult problem. This provides promise for future formulations based on other QoS constraints.

The rest of the paper is organized as follows. In Section II, we present the data flow model and the transmission model followed by the problem description. In Section III, we consider the deterministic case and present the optimal transmission policy. Section IV presents the results for the stochastic case. Finally, Section V concludes the work and gives future research directions.

\section{Problem Setup}

\section{A. Data Flow Model}

We consider a continuous time system model that incorporates both the continuous and the packetized data flow scenarios. The data flows are described using the notions of arrival and departure curves while the QoS constraints are abstracted with the new concept of a minimum departure curve. For uniformity, all functions in this paper are assumed to be right-continuous. This assumption conforms with the packetized data arrival model.

Definition 1: An arrival curve $A(t), t \geq 0, t \in \mathbb{R}$ is a rightcontinuous function defined as the number of bits that have arrived in time interval $[0, t]$.

In case of a fluid data model $A(t)$ is a continuous function, whereas, for a packet arrival model $A(t)$ is a stair-case function as depicted in Figure 1.

Definition 2: Given an arrival curve $A(t)$, a departure curve $D(t), t \geq 0, t \in \mathbb{R}$ is a right-continuous function defined as the number of bits that have departed in time interval $[0, t]$.

Similar to the arrival curve, a departure curve $D(t), t \geq 0$ is a non-decreasing function of time $t$ and satisfies the causality constraint $D(t) \leq A(t), \forall t$.

To model the QoS constraints we introduce the notion of a minimum departure curve.

Definition 3: Given an arrival curve $A(t)$, a minimum departure curve $D_{\min }(t)$ is a right-continuous function such that $D_{\text {min }}(t) \leq A(t), \forall t \geq 0$ and is defined as the minimum number of bits that must depart by time $t$ to satisfy the quality of service requirements.

Thus, $D_{\min }(t)$ is the constraint function and to meet the QoS requirements the actual departure curve $D(t)$ must satisfy $D(t) \geq D_{\min }(t)$ with the additional causality constraint
$D(t) \leq A(t), \forall t$. We, next, present a few illustrative examples of commonly used QoS constraints that can be modelled within this framework.

Delay Constraint: Consider an arrival curve $A(t)$ and a constant delay constraint $d$ on all the data. In this case $D_{\text {min }}(t)=0, t \in[0, d)$ and $D_{\text {min }}(t)=A(t-d), t \geq d$.

Next consider a continuous data flow and a general deadline function $d(t)$; where $d(t)$ is the deadline for data arriving at time $t$. Assuming that $h(s)=s+d(s)$ is a monotonically increasing function, the minimum departure curve is $D_{\min }(t)=$ $0, t \in[0, d(0))$ and $D_{\min }(t)=A\left(h^{-1}(t)\right), t \geq d(0)$. We can similarly construct $D_{\min }(t)$ for the case of packetized arrivals and variable deadline.

Buffer Constraint: Consider a buffer constraint of $B$, i.e. the queue size must not exceed $B, \forall t \geq 0$. For an arrival curve $A(t)$ and a departure curve $D(t)$ the buffer size at any time $t$ is given by $b(t)=A(t)-D(t)$. Since $b(t) \leq B$, we have $D(t) \geq \max [A(t)-B, 0]$. This gives the minimum departure curve as $D_{\min }(t)=\max [A(t)-B, 0]$. It is easy to incorporate a time varying buffer constraint $B(t)$ as well.

Service-Curve Constraint: The notion of service curves forms an integral part of network calculus theory [6] and is defined as follows.

Definition 4: Consider a system $S$ and a flow through $S$ with arrival and departure curves $A(t)$ and $D(t)$. We say that $S$ offers to the flow a service curve $\beta(t)$ if and only if $\beta(t)$ is wide sense increasing, $\beta(0)=0$ and,

$$
D(t) \geq \inf _{s \leq t}(A(s)+\beta(t-s)) \triangleq A(t) \otimes \beta(t)
$$

Given a service curve $\beta(t)$ and an arrival curve $A(t)$, the minimum departure curve can be obtained as $D_{\min }(t)=$ $A(t) \otimes \beta(t)$, where $\otimes$ is the convolution in the min-plus algebra as defined in (1).

Thus, a wide variety of QoS constraints can be abstracted by constructing the appropriate minimum departure curve.

\section{B. Transmission Model}

To each departure curve, $D(t)$, we associate an energy cost based on the following model. The power $P$ required to reliably transmit at a certain rate $r$ is assumed to be a convex increasing function of the rate, i.e. $P=g(r)$ where $g($.$) is a$ convex function such that $g(r) \geq 0$ and strictly increasing for $r \geq 0$. This assumption is common in the literature [1], [2], [3], [4], [7] and is a good model for practical coding schemes. Physically, this means that as the transmission rate increases the required power has a faster increase and hence lower rate transmissions are preferable. One of the examples that justify this claim is the Shannon capacity formula over an AWGN channel. The capacity is a concave function of the power and is given as,

$$
C=\frac{1}{2} \log \left(1+\frac{P}{N}\right) \quad \text { bits/transmission }
$$

Re-writing the above equation it easily follows that the expended power is a convex function of the transmission rate. The reader is referred to [7], [1] for other coding schemes that 
support the convexity assumption. Note that in our formulation we do not restrict ourselves to any specific function but rather assume any general convex relationship between power and transmission rate.

Using the above model we now compute the energy expenditure of departure curves that are continuous in time. For any such curve the rate of transmission at any time $t$ is its derivative at $t$, i.e. $r=D^{\prime}(t)$. If at $t$ the derivative does not exist then we take $r$ as the right-derivative at that point. This notation will be followed throughout the paper for derivatives of other functions as well ${ }^{1}$. The energy spent in time interval $[0, T]$ is then given by,

$$
\mathcal{E}(D(t))=\int_{0}^{T} g\left(D^{\prime}(t)\right) d t
$$

The average power expended is simply $\mathcal{E}(D(t)) / T$. In the rest of the paper we focus our attention over the interval $[0, T]$ for any finite $T$. Thus, we deal with energy minimization over a finite time interval rather than in an average sense over an infinite time horizon.

\section{Problem Definition}

We begin by first considering the deterministic case for which the arrival curve is known over time interval $[0, T]$. Based on the QoS requirements, one can then construct the minimum departure curve as outlined in Section II-A. We call a departure curve feasible if it satisfies both the causality and the QoS constraint; i.e. $D_{\min }(t) \leq D(t) \leq A(t), t \in$ $[0, T]$. We consider only continuous departure curves as any discontinuity would imply instantaneous transmission of nonzero amount of data which is practically infeasible. Let $\Gamma$ be the set of all non-decreasing continuous curves in $[0, T]$ that start at the origin. The energy minimization problem can now be stated as follows,

$$
\begin{aligned}
\min _{D(t)} & \mathcal{E}(D(t))=\int_{0}^{T} g\left(D^{\prime}(t)\right) d t \\
\text { subject to } & D_{\min }(t) \leq D(t) \leq A(t), t \in[0, T] \\
& D(t) \in \Gamma
\end{aligned}
$$

with the following natural conditions,

$$
\text { (a) } D_{\min }(0)=0 \text {; and (b) } D_{\min }(T)=A(T)
$$

As we are only concerned with the interval $[0, T]$, condition (b) simply states that all the data must depart by time $T$. If one makes the natural assumption that there is no data that arrives and needs to be transmitted instantaneously then there exists a solution to the above problem; i.e. a non-decreasing continuous feasible $D(t)$ exists.

The above formulation assumes that the rate can be adjusted continuously in time. This is a good mathematical model for practical systems in which the slot duration is small compared to the time scales of data flow. A departure curve specifies the transmission rate at time $t$ and hence we will use the terms departure curve and transmission policy interchangeably.

\footnotetext{
${ }^{1}$ By right-continuity of curves the right-derivative exists at all points.
}

In the latter half of the paper we introduce stochasticity into the arrival process. The general problem definition remains the same as the deterministic case with the exception that the instantaneous transmission rate is chosen to minimize the expected energy expenditure. As noted earlier, in this case, we consider a Poisson arrival process and a single deadline constraint. The mathematical formulation and the solution are presented in Section IV in a self-contained manner.

\section{Transmission Policy - Deterministic CAse}

\section{A. Optimality Criterion}

We assume that $A(t)>D_{\min }(t), 0<t<T$; otherwise say at some time $t_{e}$ there is equality then the problem can be divided into two sub-problems over time intervals $\left[0, t_{e}\right]$ and $\left[t_{e}, T\right]$. The following lemma is an integral version of Jensen's inequality and will be used later in proving the optimality criterion.

Lemma 1: Let $f(x), p(x)$ be two functions defined for $a \leq$ $x \leq b$ such that $\alpha \leq f(x) \leq \beta$ and $p(x)>0$, with $p(x) \not \equiv 0$. Let $\phi(u)$ be a convex function defined on the interval $\alpha \leq$ $u \leq \beta$; then

$$
\phi\left(\frac{\int_{a}^{b} f(x) p(x) d x}{\int_{a}^{b} p(x) d x}\right) \leq \frac{\int_{a}^{b} \phi(f) p(x) d x}{\int_{a}^{b} p(x) d x}
$$

with strict inequality if $\phi()$ is strictly convex and $a \neq b, \alpha \neq \beta$.

Proof: See [10].

The following Theorem presents the necessary condition for any feasible departure curve to be optimal.

Theorem I: (Optimality Criterion) Let $D(t) \in \Gamma$ be a feasible departure curve and $L(t)$ be a straight line segment over $[a, b]$ that joins points $D(a)$ and $D(b) ; 0 \leq a<b \leq T$. If $L(t)$ is feasible, i.e. $D_{\min }(t) \leq L(t) \leq A(t)$ and $L(t) \not \equiv D(t)$ then the new departure curve $\tilde{D}(t)$ constructed as,

$$
\begin{aligned}
\tilde{D}(t) & =D(t), t \in[0, a) \\
& =L(t), t \in[a, b] \\
& =D(t), t \in(b, T]
\end{aligned}
$$

satisfies $\mathcal{E}(\tilde{D}(t)) \leq \mathcal{E}(D(t))$ where the inequality is strict if $g($.$) is strictly convex.$

The above Theorem states that for the optimal curve $D^{\text {opt }}(t)$ there does not exist any two points on the curve that can be joined by a straight line without violating the feasibility constraints and with the straight line not being part of $D^{o p t}(t)$. The implication of this is that, if feasible, it is optimal to transmit at a constant rate. Henceforth, we refer to this as the optimality criterion.

Proof: Let $D(t)$ be a given feasible departure curve and suppose that there exists an interval $[a, b], b>a$ and feasible $L(t)$ such that $L(t) \not \equiv D(t)$; where $L(t)$ is the straight line joining $D(a)$ and $D(b)$. Construct the departure curve $\tilde{D}(t)$ as stated in the Theorem. Its clear that $\tilde{D}(t)$ is non-decreasing, continuous and feasible. The slope of $L(t)$ is $L^{\prime}=\frac{D(b)-D(a)}{b-a}$. The energy difference between $D(t)$ and $\tilde{D}(t)$ is given by,

$$
\mathcal{E}(\tilde{D}(t))-\mathcal{E}(D(t))=\int_{a}^{b} g\left(L^{\prime}\right) d t-\int_{a}^{b} g\left(D^{\prime}(t)\right) d t
$$




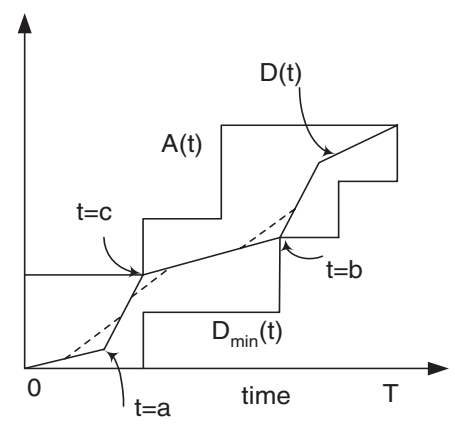

Fig. 2. Example depicting violation of Corollaries I.1-I.3. The dotted line indicates straight line segments that would violate the optimality criterion.

$$
\begin{aligned}
& =\int_{a}^{b} g\left(\frac{D(b)-D(a)}{b-a}\right) d t-\int_{a}^{b} g\left(D^{\prime}(t)\right) d t \\
& =(b-a) g\left(\frac{D(b)-D(a)}{b-a}\right)-\int_{a}^{b} g\left(D^{\prime}(t)\right) d t
\end{aligned}
$$

Using Lemma 1 with the following substitution; $p()=1$, $\phi()=g(), f()=D^{\prime}()$ and $x=t$ we get,

$$
\begin{aligned}
g\left(\frac{\int_{a}^{b} D^{\prime}(t) d t}{\int_{a}^{b} d t}\right) & \leq \frac{\int_{a}^{b} g\left(D^{\prime}(t)\right) d t}{\int_{a}^{b} d t} \\
g\left(\frac{D(b)-D(a)}{b-a}\right) & \leq \frac{\int_{a}^{b} g\left(D^{\prime}(t)\right) d t}{b-a}
\end{aligned}
$$

Combining (8) and (10) we get,

$$
\mathcal{E}(\tilde{D}(t))-\mathcal{E}(D(t)) \leq 0
$$

with strict inequality if $g($.$) is strictly convex.$

The optimality criterion suggests that transmitting at a constant rate is more energy efficient as compared to a variable rate transmission. However, a constant transmission rate cannot always be maintained due to feasibility constraints. In what follows we consider the properties of the points at which the rate changes (slope of $D^{\text {opt }}(t)$ changes). These implications fall out of Theorem I and are instrumental in constructing the optimal curve. The notation $x^{+}$means $x+\epsilon_{n}$ and $x^{-}$means $x-\epsilon_{n}$ with $\epsilon_{n}>0, \epsilon_{n} \rightarrow 0$.

Let $D^{o p t}(t)$ be the optimal departure curve and $t_{0}$ be a point at which its slope changes, i.e. $\left(D^{o p t}\right)^{\prime}\left(t_{0}^{-}\right) \neq\left(D^{o p t}\right)^{\prime}\left(t_{0}^{+}\right)$.

Corollary I.1: At $t_{0}$, the optimal curve either intersects $A(t)$ or it intersects $D_{\min }(t)$; i.e. we have $D^{\text {opt }}\left(t_{0}\right)=A\left(t_{0}\right)$ or $D^{\text {opt }}\left(t_{0}\right)=D_{\min }\left(t_{0}\right)$. Note that if there is a discontinuity in $A(t)$ at $t_{0}$ (stair-case jump for packetized data) then $D^{\text {opt }}\left(t_{0}\right)=A\left(t_{0}^{-}\right)$.

Corollary I.2: Suppose at $t_{0}$, we have $D^{\text {opt }}\left(t_{0}\right)=$ $D_{\min }\left(t_{0}\right)$ then the change in slope must be negative.

Corollary I.3: Suppose at $t_{0}$, we have $D^{o p t}\left(t_{0}\right)=A\left(t_{0}\right)$ (or $A\left(t_{0}^{-}\right)$) then the change in slope must be positive.

The proofs of the above corollaries are straightforward and omitted for brevity. They can be easily understood from Figure 2. Point $t=a$ corresponds to a violation of Corollary I.1 and it is easy to see that around $t=a$, the optimality criterion is violated. Similarly, points $t=b$ and $t=c$ correspond to a violation of Corollaries I.2 and I.3 respectively.

Remark 1: In the special case when the power-rate relationship is linear, i.e. $P=c r$, it follows from Theorem I that all feasible departure curves have the same energy expenditure as the inequality in Lemma 1 becomes an equality. Henceforth, we consider the more interesting case of strictly convex $g()$.

The following theorem proves the uniqueness of $D^{\text {opt }}(t)$ when $g($.$) is strictly convex. Combined with Theorem I this$ shows that the optimality criterion is necessary and sufficient.

Theorem II: (Uniqueness) Consider the optimization problem stated in (4) with the boundary condition in (5) and $g($.$) being strictly convex. Then, the optimal departure curve$ $D^{o p t}(t)$ is unique.

Proof: Let us assume that the optimal departure curve is not unique. Let $D_{1}(t)$ and $D_{2}(t)$ be two distinct optimal curves. By the assumption of optimality both these curves must satisfy the optimality criterion and Corollaries I.1-I.3. We also have the boundary conditions $D_{1}(0)=D_{2}(0)=0$ and $D_{1}(T)=D_{2}(T)=D_{\min }(T)$. Since $D_{1}(t) \not \equiv D_{2}(t)$ the two curves must differ over some time interval in $[0, T]$. Let $t=a$ be the first instant after which the two curves differ and $t=b$ be the first time instant after $t=a$ at which they are equal again. Note that $b \leq T$ as at time $T, D_{1}(T)=D_{2}(T)$. Without loss of generality let $D_{1}(t)>D_{2}(t), t \in(a, b)$. From the feasibility of the two curves we have,

$$
D_{\text {min }}(t) \leq D_{2}(t)<D_{1}(t) \leq A(t), t \in(a, b)
$$

As $D_{1}(t)$ is strictly greater than $D_{\min }(t)$ in $t \in(a, b)$ it follows from Corollaries I.1-I.3 that its slope cannot decrease in $(a, b)$. This implies that $D_{1}(t)$ is convex (it could be linear as well) in $(a, b)$. Similarly as $D_{2}(t)$ is strictly less than $A(t)$ in $t \in(a, b)$, its slope cannot increase and hence it must be concave in $(a, b)$. Its clear that $D_{1}(t)$ convex and $D_{2}(t)$ concave in $t \in(a, b)$ (and $D_{1}(a)=D_{2}(a)$ ) cannot be equal again at $t=b$ which leads to a contradiction. Finally, if both curves are linear in $(a, b)$ (with equality at $t=a, t=b)$ then it would violate the assumption $D_{1}(t) \neq D_{2}(t), t \in(a, b)$.

We next present a few interesting properties of the optimal curve. Among the set of $D(t) \in \Gamma$ that are feasible, the optimal departure curve is such that it requires the least maximum transmission power and has the smallest Euclidean length.

Theorem III: (Minimal Maximum Power) Consider the optimization problem in (4) with the boundary condition in (5) and $g($.$) being strictly convex. The optimal departure curve$ $D^{o p t}(t)$ satisfies,

$$
\max _{t \in[0, T)}\left(D^{o p t}\right)^{\prime}(t) \leq \max _{t \in[0, T)} D^{\prime}(t)
$$

Equivalently, $\max _{t \in[0, T)} P^{o p t}(t) \leq \max _{t \in[0, T)} P(t)$, where $P($.) denotes the power expenditure over time.

Proof: See Appendix II

Remark 2: The above theorem is very significant if we impose an additional maximum power constraint in (4). In this case we first solve the problem without the power constraint. If the optimal solution satisfies the maximum power constraint 


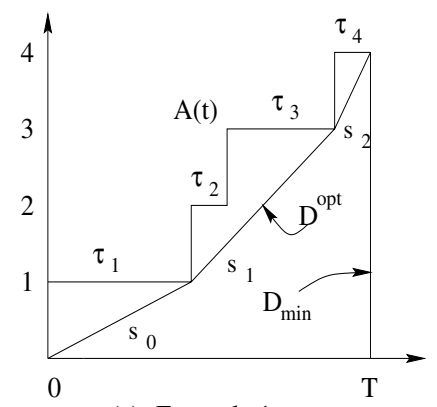

(a)- Example 1

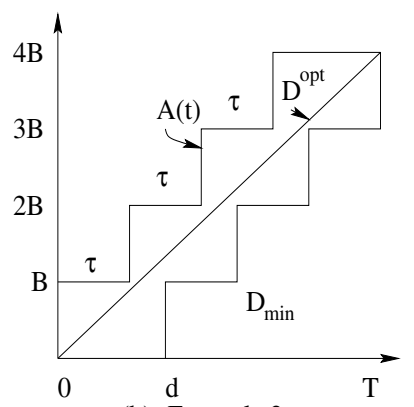

(b)- Example 2
Fig. 3. The curves $A(t), D_{\min }(t), D^{o p t}(t)$ for Examples 1 and 2 .

then we are done; otherwise from Theorem III it follows that there does not exist any other feasible departure curve that can satisfy the power constraint and the constrained optimization problem has no solution. Thus, this implies that $D^{o p t}(t)$ is the unique curve that satisfies the QoS constraints with the least average power and the least maximum power requirement.

Theorem IV: (Shortest Length) Consider the optimization problem in (4) with the boundary condition in (5) and $g($. being strictly convex. The optimal departure curve $D^{\text {opt }}(t)$ has the shortest length among feasible curves. Specifically it minimizes the metric,

$$
\operatorname{len}(D(t)) \triangleq \int_{0}^{T} \sqrt{\left(1+\left(D^{\prime}(t)\right)^{2}\right)} d t
$$

Proof: We know that $D^{o p t}(t)$ minimizes the integral in (3) for any strictly convex increasing function $g($.$) . As$ $\sqrt{\left(1+\left(D^{\prime}(t)\right)^{2}\right)}$ is a strictly convex increasing function, the result follows by replacing $g($.$) with this function.$

Remark 3: Based on the properties of $D^{o p t}(t)$ we have the following very insightful and intuitive visualizations of the optimal curve. First, it is the shortest length feasible continuous curve between 0 and $D_{\min }(T)$ as outlined in Theorem IV. Second, consider a string that is restricted to lie between $A(t)$ and $D_{\min }(t)$. Tie its one end at the origin and pass the other end through $D_{\min }(T)$. Now if we make the string taut, its trajectory is the same as the optimal curve ${ }^{2}$.

\section{B. Examples}

Before we present the mathematical details of the optimal policy, it is instructive to consider a few illustrative scenarios that can be modelled within this framework. The solutions to these examples can be understood based on the optimality criterion and the intuitive visualization in Remark 3. Some of these examples have been studied earlier in the literature for which the solutions were obtained using classical discrete optimization techniques. Here, we re-formulate those problems as special cases to our general solution.

Example 1: This problem was studied in [1] and the optimal offline solution was obtained using discrete convex optimization approach. Consider $N$ packets of unit size arriving in time $[0, T)$ with known inter-arrival times $\tau_{1}, . ., \tau_{N-1}$ and

\footnotetext{
${ }^{2}$ This observation was pointed out by Rene L. Cruz
}

the first packet arrival at time 0 . The $\tau_{i}^{\prime} s$ are such that $\tau_{1}+. . .+\tau_{N-1}<T$ and all packets must depart by time $T$ (common deadline). Let $\tau_{N}=T-\sum_{1}^{N-1} \tau_{i}$. The curves $A(t)$ and $D_{\min }(t)$ for this problem are depicted in Figure 3(a). From the optimality criterion and the string interpretation it is easy to see that the optimal curve consists of piecewise linear segments with increasing slopes. At points where the slope changes, the optimal policy just empties the buffer. The intuition is that the optimal policy attempts to transmit at a constant rate subject to causality constraints. It empties the buffer at points (of slope change) where the future arrivals are such that relative to the deadline the transmission rate must be higher. It can be directly verified that such a curve satisfies the optimality criterion. Algebraically, the slopes of the linear segments $\left\{s_{0}, . ., s_{l}\right\}$ can be computed recursively as follows. Let $k_{0}=0$, then,

$$
\begin{aligned}
s_{m} & =\min _{j \in\left\{1, . ., N-k_{m}\right\}} \frac{j}{\sum_{i=1}^{j} \tau_{i+k_{m}}} \\
k_{m+1} & =k_{m}+\arg \min _{j \in\left\{1, . ., N-k_{m}\right\}} \frac{j}{\sum_{i=1}^{j} \tau_{i+k_{m}}}
\end{aligned}
$$

The final segment is the one for which $k_{m+1}=N$.

Example 2: Consider a stream of $N$ regular packet arrivals of size $B$ and constant inter-arrival time $\tau$. Each packet has a deadline $d$ before which it must depart (Figure 3(b)). Such an arrival process closely models the voice traffic and other applications that send data at regular intervals. The solution is obvious from the figure and is given as follows. If $d<\tau$ then the solution is trivial and the packet must be transmitted before the next arrival. If $d \geq \tau$ then the optimal curve is a straight line with slope $N B /(d+(N-1) \tau)$. Thus the optimal policy in this case is to transmit at a constant rate and the rate is chosen such that feasibility constraints are met.

Example 3: This problem was considered in [3]. We generalize it so that the deadlines need not be integral multiples and re-formulate it with our framework. Consider an amount $B$ of data in the buffer at time 0 and no new arrivals. Let the data have discrete deadlines with $b_{k}$ amounts having deadline $d_{k}$, for $k=1, . ., N ; d_{N}=T$ and $\sum_{1}^{N} b_{i}=B$; the data being arranged in the earliest deadline first order. The curves $A(t)$ and $D_{\min }(t)$ for this system are shown in Figure 4(a). From the figure it is clear that the optimal curve consists of linear segments with decreasing slopes as depicted in the figure. Note, the opposite nature of this problem as compared to Example 1. In this case the points at which the slope changes the optimal curve just meets the deadline constraints. Algebraically, the slopes $\left\{s_{0}, . ., s_{l}\right\}$ can be obtained as follows. Let $\gamma_{k}=d_{k}-d_{k-1}, k=2, . ., N ; \gamma_{1}=d_{1}$ and $k_{0}=0$.

$$
\begin{aligned}
s_{m} & =\max _{j \in\left\{1, . ., N-k_{m}\right\}} \frac{\sum_{i=1}^{j} b_{i+k_{m}}}{\sum_{i=1}^{j} \gamma_{i+k_{m}}} \\
k_{m+1} & =k_{m}+\arg \max _{j \in\left\{1, . ., N-k_{m}\right\}} \frac{\sum_{i=1}^{j} b_{i+k_{m}}}{\sum_{i=1}^{j} \gamma_{i+k_{m}}}
\end{aligned}
$$

At the last segment $k_{m+1}=N$.

Example 4: Consider continuous arrival and minimum departure curves as shown in Figure 4(b). The optimal departure 

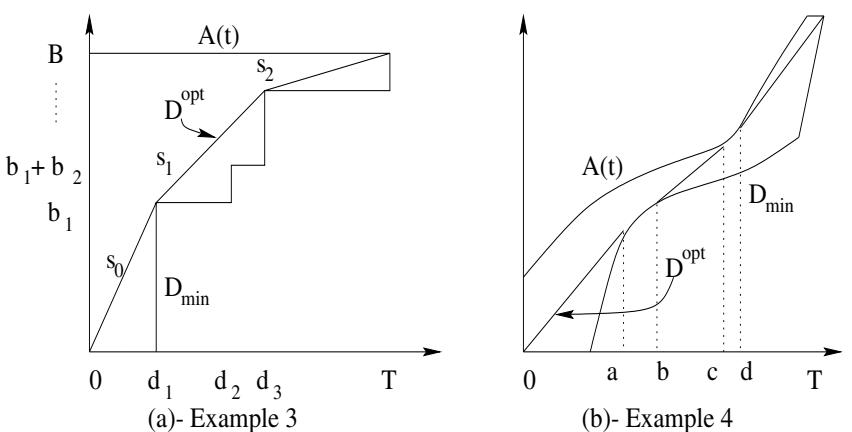

(b)- Example 4

Fig. 4. The curves $A(t), D_{\min }(t), D^{o p t}(t)$ for Examples 3 and 4 .

curve is shown in the figure and it consists of some segments that have a continuously changing slope.

We now complete the discussion of the known arrival case with a mathematical description of the algorithm to construct the optimal curve.

\section{Construction of the Optimal Policy}

In this section, we present an iterative algorithm to obtain the optimal curve. To describe this algorithm we need a few properties that are presented next.

Definition 5: A tangent to $D_{\min }(t)$ at $t=t_{0}$ is a line passing through $\left(t_{0}, D_{\min }\left(t_{0}\right)\right)$ and slope $D_{\min }^{\prime}\left(t_{0}\right)$.

A similar definition follows for a tangent to $A(t)$. Next, we need the notion of intersection of curves. As the arrival model also includes stair-case functions (packet arrivals) that have discontinuities we need to define what it means to intersect such curves. This is done next.

Consider a line $L(t)$ of non-negative slope starting from a feasible point $\left(t_{0}, \alpha\right)$; where feasibility of a point means $0 \leq t_{0}<T$ and $D_{\min }\left(t_{0}\right) \leq \alpha \leq A\left(t_{0}\right)$.

Definition 6: We say that beyond $t_{0}, L(t)$ intersects $D_{\min }(t)$ if for some point $\tilde{t}>t_{0}$, called the point of intersection, one of the following holds; (a) either $L(\tilde{t})=$ $D_{\min }(\tilde{t})$ or, (b) the function $L(t)-D_{\min }(t)$ changes sign at $\tilde{t}$ (here $\tilde{t}$ could be a discontinuity point).

Definition 7: We say that $L(t)$ intersects $D_{\min }(t)$ first if $L(t)$ intersects $D_{\min }(t)$ at $\tilde{t}\left(>t_{0}\right)$ and $L(t)<A(t), t \in$ $\left(t_{0}, \tilde{t}\right)$ (that is, $L(t)$ does not intersect $A(t)$ in $\left(t_{0}, \tilde{t}\right)$ ).

Similarly, we say that $L(t)$ intersects $A(t)$ first if $L(t)$ intersects $A(t)$ at $\tilde{t}$ and $L(t)>D_{\min }(t), t \in\left(t_{0}, \tilde{t}\right)$. Now, to obtain the optimal curve, we need to figure out what the optimal slope should be starting at some feasible point. We proceed next to obtain this slope.

Consider straight lines with non-negative slopes passing through a feasible point $\left(t_{0}, \alpha\right)$. Among these choose those lines that starting at $\left(t_{0}, \alpha\right)$ remain feasible for some finite duration ${ }^{3}$ and denote this set as $\mathcal{F}$. Note that the set $\mathcal{F}$ depends on the starting point $\left(t_{0}, \alpha\right)$ but to make the notations simple we drop the explicit dependence. The following lemmas summarize the properties of set $\mathcal{F}$.

\footnotetext{
${ }^{3}$ There exists $\epsilon>0$ such that the straight line is feasible in $t \in\left[t_{0}, t_{0}+\epsilon\right)$.
}

Lemma 2: The slopes of the lines in $\mathcal{F}$ lie in a continuous interval.

Proof: See Appendix IV.

The set $\mathcal{F}$ has the following possibilities. For $A\left(t_{0}\right)>$ $D_{\min }\left(t_{0}\right)$ we have the following three cases (i) If $D_{\min }\left(t_{0}\right)<$ $\alpha<A\left(t_{0}\right)$ then due to right continuity of the curves all points in a small region around $\alpha$ are feasible. Hence, all lines with slopes lying in $[0, \infty)$ are feasible for some finite duration starting at $\left(t_{0}, \alpha\right)$. (ii) If $\alpha=D_{\min }\left(t_{0}\right)$, all lines with slope less than the tangent at $D_{\min }\left(t_{0}\right)$ (say slope $c$ ) are infeasible while lines with slope greater than the tangent are feasible for some finite duration. If the tangent itself is feasible then the slopes of $\mathcal{F}$ lie in $[c, \infty)$; else they lie in $(c, \infty)$. (iii) If $\alpha=A\left(t_{0}\right)$ then lines with slopes less than the tangent at $A\left(t_{0}\right)$ belong to $\mathcal{F}$. If the tangent is feasible then the slopes of $\mathcal{F}$ lie in $[0, l]$; else the slopes belong to $[0, l)$.

Finally, at $t_{0}=0$ if we have $A(0)=D_{\min }(0)$, the set $\mathcal{F}$ consists of lines with slopes lying between the tangents to each curve.

Lemma 3: The lines in $\mathcal{F}$ must intersect $A(t)$ first or intersect $D_{\min }(t)$ first.

Proof: The proof is straightforward and omitted for brevity.

Let the set $\mathcal{F}$ be partitioned into a set of lines that intersect $A(t)$ first and those that intersect $D_{\min }(t)$ first. Denote these sets as $\mathcal{F}_{A}$ and $\mathcal{F}_{D_{m}}$ respectively. The following lemma states that the slopes of the lines in $\mathcal{F}_{A}$ and $\mathcal{F}_{D_{m}}$ lie in nonoverlapping continuous intervals.

Lemma 4: (a) Let $L_{D}(t) \in \mathcal{F}_{D_{m}}$ then any $L(t) \in \mathcal{F}$ that has slope less than $L_{D}^{\prime}$ intersects $D_{\min }(t)$ first. (b) Let $L_{A}(t) \in \mathcal{F}_{A}$ then any $L(t) \in \mathcal{F}$ that has slope greater than $L_{A}^{\prime}$ intersects $A(t)$ first.

Proof: See Appendix IV.

The above lemma has the following implications. First, the slopes of the lines in $\mathcal{F}_{A}$ and $\mathcal{F}_{D_{m}}$ lie in non-overlapping continuous intervals which we denote as $\mathcal{S}_{A}$ and $\mathcal{S}_{D_{m}}$ respectively. Second, the slopes in $\mathcal{F}_{A}$ are greater than in $\mathcal{F}_{D_{m}}$. The optimal line is the one with slope $\beta_{o}$ at the boundary of the two intervals ${ }^{4}$; i.e. $\beta_{o}$ is given as,

$$
\beta_{o}=\inf \mathcal{S}_{A}=\sup \mathcal{S}_{D_{m}}
$$

The equality of inf and sup above follows from continuity as in Lemma 2. If either $\mathcal{S}_{A}$ or $\mathcal{S}_{D_{m}}$ is empty, it is neglected. We call $\beta_{o}$ the optimal slope and the line with slope $\beta_{o}$ the optimal line. It is denoted as $L_{o}$. The line $L_{o}$ forms the building block of the optimal departure curve as illustrated later in the algorithm. Since $\beta_{o}$ is unique, $L_{o}$ is also unique which conforms with the result that the optimal curve is unique.

\section{Optimal Policy}

We have $D^{o p t}(0)=0$ and $D^{\text {opt }}(T)=D_{\min }(T)$. We know that the slope of $D^{o p t}(t)$ changes at specific points (Corollaries

\footnotetext{
${ }^{4} \mathrm{~A}$ case of singularity occurs at $t_{0}=0$ if $A(0)=D_{\min }(0), A^{\prime}(0)=$ $D_{\text {min }}^{\prime}(0)$ and both the sets $\mathcal{S}_{A}, \mathcal{S}_{D_{m}}$ are empty. Here, simply define $\beta_{o}=$ $A^{\prime}(0)$.
} 


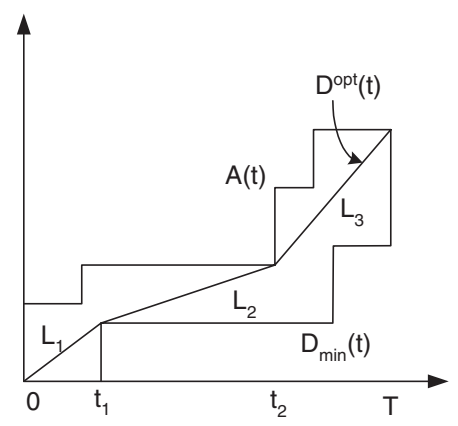

Fig. 5. Example depicting $A(t), D_{\min }(t)$ and the constructed $D(t)$.

I.1-I.3), hence, we can compute the segments of $D^{\text {opt }}(t)$ in a recursive fashion starting at $(0,0)$. Let us denote $t_{0}$ as a generic starting time.

1) Obtain $\beta_{o}$ as in (19) and the optimal line $L_{o}$. This can be done by a bi-sectioning algorithm.

2) If $L_{o}$ is not tangent to $D_{\min }(t)$ (or $A(t)$ ) at $t_{0}$ then obtain the first instant $t_{1}$ such that, (a) $L_{o}\left(t_{1}\right)=D_{\min }\left(t_{1}\right)$ (constraint is just met); or (b) $L_{o}\left(t_{1}\right)=A\left(t_{1}\right)$ or $L_{o}\left(t_{1}\right)=A\left(t_{1}^{-}\right)$(buffer is just empty). Let $D^{o p t}(t)=$ $L_{o}(t), t \in\left(t_{0}, t_{1}\right]$. Note that $t_{1}$ is the first instant on $L_{o}$ at which the slope can change in accordance with Corollary I.1.

3) If $L_{o}$ is tangent to $D_{\min }(t)$ (or $A(t)$ ) at $t_{0}$ then let $t_{1}=\min \{\tilde{t}, T\}$ where $\tilde{t}$ is the first instant at which the tangent is no more the optimal line. Let $D^{o p t}(t)=$ $D_{\min }(t)$ (or $\left.A(t)\right), t \in\left(t_{0}, t_{1}\right]$.

If $t_{1}=T$ terminate; else repeat the above steps with the new starting point as $\left(t_{1}, D^{o p t}\left(t_{1}\right)\right)$. The correctness and optimality of the above algorithm is shown in Appendix I.

Consider $A(t)$ and $D_{\min }(t)$ shown in Figure 5 for which the algorithm executes as follows. Start at the origin $(0,0)$ and note that $L_{1}$ is the optimal line as defined above and $t_{1}$ is the first instant at which it equals $D_{\min }(t)$. Thus, segment $L_{1}$ from $t=\left[0, t_{1}\right]$ is the first part of the optimal curve. Note that lines with slope $>L_{1}^{\prime}$ intersect $A(t)$ first and lines with slope $<L_{1}^{\prime}$ intersect $D_{\min }(t)$ first. $L_{1}$ is the line with slope at the boundary (as defined in (19)). Next, starting from the new point $\left(t_{1}, D_{\min }\left(t_{1}\right)\right), L_{2}$ is the optimal line and $t_{2}$ is the first instant such that $L_{2}\left(t_{2}\right)=A\left(t_{2}^{-}\right)$. The segment $L_{2}$ from $t=\left[t_{1}, t_{2}\right]$ forms part of the optimal curve. Finally, $L_{3}$ is the last segment as $t=T$ is reached. Consider next the example shown in Figure 4(b). Here, the first line segment terminates at $t=a$. Then, starting at $t=a$, the tangent to $D_{\min }(t)$ is the optimal line and $t=b$ is the first instant at which the tangent is no more the optimal line. Thus, here we get a continuously changing slope of $D^{o p t}(t)$ in $t \in(a, b)$. Along similar lines we have $D^{o p t}(t)=A(t)$ in $t \in[c, d]$. It can be easily verified that in both the examples above, the constructed curve satisfies the optimality criterion and the interpretation in Remark 3.

Using the intuition gained from the optimal solution in the off-line known arrival case one can easily construct application specific heuristic online policies when the future arrivals are unknown. We present below one such policy for packetized data model.

Online Policy: This policy is based on the solution of Example 3 presented earlier. Let $t$ be a packet arrival instant then the buffer at $t+$ (immediately after the packet arrival) consists of (a) earlier packets with their deadlines and (b) the new packet with its own deadline. Re-arranging the data in the Earliest-deadline first order, we can view the buffer as consisting of some total data $B$ with different deadlines. This is identical to Example 3 and hence we can obtain an optimal solution. This optimal policy is followed until a new arrival occurs at which point the optimization is re-done.

The above heuristic policy does not take into account any knowledge of the arrival statistics and its obvious that including such information will only improve the performance. In the next section we present a formulation with stochastic arrivals and obtain an online policy. The simulation results indeed show very significant improvements when such statistical information is included.

\section{TRansmission Policy - Stochastic CASE}

We have, until now, obtained the optimal policy for transmitting data with a known arrival and minimum departure curve. Now, we extend the formulation to a stochastic arrival process. We consider a Poisson arrival data stream with a single deadline by which all the data must be transmitted. This problem is the stochastic version of Example 1 where the unknown arrival curves are now the sample paths of the Poisson process. The problem models a system offering service with a convex cost over a finite duration by which the unfinished work must be zero; for example, a polling system [11] where a central entity polls individual transmitters for finite durations during which the transmitter empties its buffer and any new arrivals.

\section{A. Problem Definition}

Consider a queue with a Poisson arrival process of rate $\lambda$ and packet size $B$ over time interval $[0, T)$. The arrivals occur in time $[0, T)$ and the deadline constraint is that all this data must depart by time $T+\tau_{0}$; where $\tau_{0}>0$ is fixed. There are no arrivals in $\left[T, T+\tau_{0}\right]$ and any remaining data at time $T$ is simply transmitted at a constant rate over the next $\tau_{0}$ duration. Thus in terms of buffer occupancy, the constraint is that the buffer size must be zero at time $T+\tau_{0}$.

Let the system state be defined as $(x, t)$; where time $t$ is included as an explicit variable and $x$ is the buffer size at time $t$. Let $r_{t}$ be the controllable transmission rate at time $t$. We say that a policy $r_{t}$ is feasible if $r_{t} \geq 0$ and $x \geq 0, \forall t \in[0, T]$. These are natural constraints and imply that the transmission rate and the buffer size must be non-negative. As the rate can be adjusted continuously, starting from a non-negative buffer, the constraint $x(t) \geq 0$ can be achieved by setting $r_{t}=0$ if $x(t)=0$. Thus, for any feasible policy the state space is the region $x \geq 0, t \in[0, T]$. The two boundaries of this region are $(x=0,0 \leq t<T)$ and $(x \geq 0, t=T)$. Let us denote the interior of this region as $G ; G \equiv(x>0,0 \leq t<T)$ 
and let $\partial G$ denote its two above stated boundaries. It can be shown that among the set of measurable feasible policies the optimal policy is Markov, i.e. it depends only on the present state of the system. This follows from the memoryless property of the Poisson process. Hence, we will consider only Markov policies which correspond to a map of the region $G$ onto $\mathbb{R}^{+}$, i.e. the transmission rate depends only on the present state $(x, t)$ and we will denote such a policy as $r(x, t)$. Under policy $r(x, t)$, the buffer $x$ at time $t$ evolves as a poisson stochastic differential equation (SDE) and is given as,

$$
d x=-r(x, t) d t+B d q
$$

The above equation ${ }^{5}$ can be understood by viewing $d x$ as the change in the buffer size over a small interval $d t$. The term $d q$ is the poisson differential and can be viewed as equal to 1 with probability $\lambda d t$, in which case $B$ gets added to the buffer, and 0 with probability $1-\lambda d t$.

Let $g(r)$ be the amount of power required to reliably transmit at rate $r$. Then, for any feasible policy $r(x, t)$ the expected energy cost is,

$$
J_{r}\left(x_{0}\right)=E\left[\int_{0}^{T} g(r(x, t)) d t+\tau_{0} g\left(\frac{x(T)}{\tau_{0}}\right)\right]
$$

where $x_{0}$ is the buffer size at time 0 and $\tau_{0} g\left(x(T) / \tau_{0}\right)$ is the terminal energy cost for the data remaining in the buffer at $T$. The optimization problem is to obtain the minimum expected energy expenditure over feasible policies and a feasible $r^{*}(x, t)$ that attains the minimum.

$$
J\left(x_{0}\right)=\min _{r(x, t)} J_{r}\left(x_{0}\right), r(x, t) \text { feasible }
$$

We assume $g($.$) to be strictly convex, smooth differentiable,$ strictly increasing function and $g(r) \geq 0, \forall r \geq 0$.

\section{B. Optimality Equations}

A classical approach to the optimization in (22) is to discretize the system, partition the time interval $[0, T]$ and apply dynamic programming (DP). However, the reader can easily verify that while one might be able to formally write down the discrete-time DP recursion, it cannot be solved analytically. Any numerical solution would be parameter specific and would not provide much insights into the problem. Thus, here we take a continuous time approach based on the theory of stochastic integration which corresponds to taking the partition size to zero. For a rigorous treatment of the topic see [12], [13], [14].

We, now, digress briefly and present the concept of a differential generator for a Markov process. Consider a stochastic process, $X_{t}$, then the differential generator of the process is defined as,

$$
L f(x, t)=\lim _{h \downarrow 0} \frac{E\left[f\left(X_{t+h}, t+h\right)\right]-f(x, t)}{h}
$$

where the expectation is conditioned on $X_{t}=x$ and $f()$ is any general function for which the limit exists. The differential

\footnotetext{
${ }^{5}$ It is a formal representation of $x(t)=x(0)+\int_{0}^{t}-r\left(x, \tau^{-}\right) d \tau+\int_{0}^{t} B d q$ where the integral is defined path-wise. See [13], [14] chap 1.
}

generator can be understood as the average time rate of change of the function $f\left(X_{t}, t\right)$. It is a natural generalization of the ordinary time derivative. For the process that follows the SDE in (20), the differential generator can be computed ([12], [13] chap 3) and is given as,

$$
\begin{aligned}
L f(x, t)=\frac{\partial f(x, t)}{\partial t} & -r(x, t) \frac{\partial f(x, t)}{\partial x} \\
& +\lambda\{f(x+B, t)-f(x, t)\}
\end{aligned}
$$

Coming back to the optimization problem in (22), let $J(x, t)$ be the optimal energy expenditure starting at time $t$ with buffer size $x$. The function $J()$ can be viewed as the cost-to-go function and can be written as,

$$
J(x, t)=\min _{r_{t}} E\left[\int_{t}^{T} g\left(r\left(x_{\tau}, \tau\right)\right) d \tau+\tau_{0} g\left(\frac{x(T)}{\tau_{0}}\right)\right]
$$

Under some technical requirements and differentiability of $J(x, t)^{6}$ it can be shown that $J(x, t)$ is the solution of the Hamilton-Jacobi-Bellman (HJB) equation ([12]-chap.4, Theorem 2, 5) with the appropriate boundary conditions. Conversely, a smooth differentiable (and uniformly integrable) solution of the HJB equation satisfying the boundary conditions is the optimal cost function. Specializing this to our problem, the optimal cost function $J(x, t)$ and the optimal rate $r^{*}(x, t)$ satisfy the following equation,

$$
\min _{r}\{g(r)+L J(x, t)\}=0, \forall(x, t) \in G
$$

where $L J()$ is the differential generator as given in (24), i.e.

$$
\begin{aligned}
L J(x, t)=\frac{\partial J(x, t)}{\partial t} & -r(x, t) \frac{\partial J(x, t)}{\partial x} \\
& +\lambda\{J(x+B, t)-J(x, t)\}
\end{aligned}
$$

and $r^{*}(x, t)$ is the value that minimizes the expression in (26). First, the constraint $r \geq 0$ can be neglected as by strict convexity of $g($.$) , it follows that r^{*}(x, t)>0$, if $x>0$. Second, the minimizing $r$ in (26) is unique as $g(r)-r \frac{\partial J(x, t)}{\partial x}$ is strictly convex for fixed $(x, t) \in G$.

To understand why (26) must be satisfied by the optimal cost function for $(x, t) \in G$, we present below a heuristic proof based on the dynamic programming equation and then making the step size tend to zero.

Heuristic Proof of (26): Consider the system described in Section IV-A and consider a uniform partition of $[0, T]$ with step size $d t$. Then, over an interval $[t, t+d t]$ the dynamic programming equation gives,

$$
\begin{gathered}
J(x, t)=\min _{r}\{g(r) d t+(1-\lambda d t) J(x-r d t, t+d t) \\
+\lambda d t J(x-r d t+B, t+d t)\}
\end{gathered}
$$

Assuming $J(x, t)$ is differentiable, we can take a first order Taylor series expansion. Collecting the terms in $d t$ we get,

$$
\begin{aligned}
\min _{r}\{ & g(r) d t+\left(-r \frac{\partial J(x, t)}{\partial x}+\frac{\partial J(x, t)}{\partial t}\right) d t \\
& +\lambda d t(J(x+B, t)-J(x, t))+o(d t)\}=0
\end{aligned}
$$

\footnotetext{
${ }^{6}$ For this problem these include all practical policies of interest.
} 
Dividing by $d t$ and taking the limit $d t \rightarrow 0$ gives (26).

Now, consider the boundary conditions on $J(x, t)$, i.e. when $(x, t) \in \partial G$. For the boundary $(x=0,0 \leq t<T)$ we get the following condition from the analysis in Appendix III,

$$
g(0)+\frac{\partial J(0, t)}{\partial t}+\lambda\{J(B, t)-J(0, t)\}=0
$$

The boundary condition for $(x, T)$ is simply the terminal energy cost over $\left[T, T+\tau_{0}\right]$ and is given as,

$$
J(x, T)=\tau_{0} g\left(x / \tau_{0}\right)
$$

Thus, $J(x, t)$ satisfies (26) with the boundary conditions (30) and (31) and $r^{*}(x, t)$ is the value that attains the minimum in (26) for a given $(x, t) \in G$. Applying the first order condition for the minimum in (26) we get,

$$
\left.\frac{\partial}{\partial r}(g(r)+L J(x, t))\right|_{\left\{r=r^{*}\right\}}=0
$$

Using the differential generator of (27) and simplifying gives,

$$
g^{\prime}\left(r^{*}\right)=\frac{\partial J(x, t)}{\partial x}
$$

Thus $r^{*}=g^{-1}\left(\frac{\partial J(x, t)}{\partial x}\right)$ and substituting back in (26) we can eliminate $r^{*}$ and obtain a partial differential equation involving a single function $J(x, t)$. Collecting all the above expressions the optimality equations for $(x, t) \in G$ with the boundary conditions can now be summarized as,

$$
\begin{aligned}
& g^{\prime}\left(r^{*}\right)=\frac{\partial J(x, t)}{\partial x} \\
& g\left(r^{*}\right)+\left.L J(x, t)\right|_{r=r^{*}}=0 \\
& g(0)+\frac{\partial J(0, t)}{\partial t}+\lambda(J(B, t)-J(0, t))=0, t \in[0, T \bigvee 36 \\
& J(x, T)=\tau_{0} g\left(\frac{x}{\tau_{0}}\right), x \geq 0
\end{aligned}
$$

\section{Constraint Relaxation}

The optimality equations, (34)-(37), apply to any general strictly convex differentiable function $g($.$) . These equations$ can be solved numerically using a finite difference method as outlined in Section IV-D. For a rigorous treatment of numerical methods to solve stochastic control problems see [14]. Our aim in this section is to obtain a closed form analytical solution and point out the significant insights into the problem.

We specialize to the case $g(r)=\alpha^{r}-1, \alpha>1^{7}$. We also consider a relaxation of the problem by eliminating the boundary condition of (36). In terms of the original problem this relaxation corresponds to ignoring the non-negativity constraints on $x(t)$ and $r_{t}$. Thus, the relaxed solution (which would be optimal for a system without such constraints) is infeasible for the original problem but it can be made feasible by setting $r(x, t)=0$, if $x=0$. In Section IV-D (Fig. 7) we compare this solution to the optimal solution obtained by

\footnotetext{
${ }^{7}$ This function with a constant multiplicative factor also models the Shannon result of (2). A constant multiplicative factor to $g(r)$ does not affect the optimal rate but simply scales the optimal cost function $J(x, t)$.
}

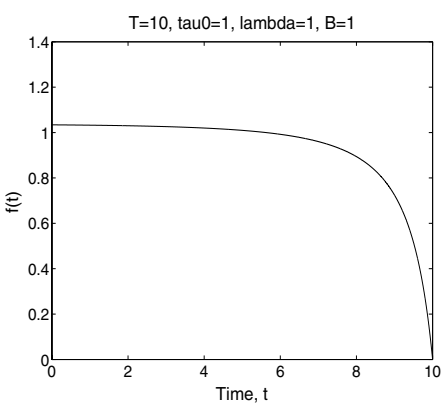

Fig. 6. Plot of $f(t)$ for $T=10, \tau_{0}=1, \lambda=1, B=1$ and $g(r)=e^{r}-1$.

numerically solving (34)-(37) and show that the policy indeed performs very close to the optimal solution.

We now proceed to solve the optimality equations without the boundary condition (36) and with $g(r)=\alpha^{r}-1, \alpha>1$. Let us take the solution $r(x, t)$ as,

$$
r(x, t)=\frac{x}{T+\tau_{0}-t}+f(t)
$$

where $f(t)$ is a function that needs to be determined. Substituting this in (34) and integrating we get,

$$
J(x, t)=\alpha^{f(t)}\left(T+\tau_{0}-t\right) \alpha^{\frac{x}{T+\tau_{0}-t}}+c(t)
$$

where $c(t)$ is the constant of integration that depends on $t$. Incorporating the boundary condition in (37) we get,

$$
f(T)=0 \text { and } c(T)=-\tau_{0}
$$

Next, substituting $J(x, t)$ in (35) and simplifying we get,

$$
\begin{gathered}
c^{\prime}(t)-1+\alpha^{f(t)}\left(T+\tau_{0}-t\right) \ln (\alpha) \alpha^{\frac{x}{T+\tau_{0}-t}} \\
\times\left\{f^{\prime}(t)-\frac{f(t)}{T+\tau_{0}-t}+\frac{\lambda}{\ln (\alpha)}\left(\alpha^{\frac{B}{T+\tau_{0}-t}}-1\right)\right\}=0
\end{gathered}
$$

Since the above equation holds for all values of $x$ the coefficients must equate to zero. Thus we get the following set of ordinary differential equations (ODE).

$$
\begin{gathered}
c^{\prime}(t)=1 \\
f^{\prime}(t)-\frac{f(t)}{T+\tau_{0}-t}+\frac{\lambda}{\ln (\alpha)}\left(\alpha^{\frac{B}{T+\tau_{0}-t}}-1\right)=0
\end{gathered}
$$

Combining (42) and (40) we get $c(t)=t-T-\tau_{0}$ while $f(t)$ can be obtained from the following lemma.

Lemma 5: Let $f(t)$ satisfy the ODE as in (43) and the boundary condition $f(T)=0$ then denoting $\beta_{t}=T+\tau_{0}-t$ we can write $f(t)$ as,

$$
\begin{aligned}
f(t) & =\frac{\lambda / \ln (\alpha)}{\beta_{t}}\left(B \ln (\alpha)(T-t)+\frac{(B \ln (\alpha))^{2}}{2} \ln \left(\frac{\beta_{t}}{\tau_{0}}\right)\right) \\
& +\frac{\lambda / \ln (\alpha)}{\beta_{t}}\left(\sum_{n=3}^{\infty} \frac{(B \ln (\alpha))^{n}}{n !(n-2)}\left(\frac{1}{\tau_{0}^{n-2}}-\frac{1}{\beta_{t}^{n-2}}\right)\right)
\end{aligned}
$$

Proof: Appendix IV

The plot of function $f(t)$ for $g(r)=e^{r}-1, T=10, \tau_{0}=1$, $\lambda=1$ and $B=1$ is shown in Figure 6. The solution thus obtained by combining (44) and (38) is an optimal solution for the problem without the non-negativity constraints on $r_{t}$ and $x(t)$. It satisfies $r(x, t)>0$, if $x>0$ but does not satisfy 
$r(x, t)=0$, if $x=0$. However, a feasible solution can be easily constructed as follows.

$$
\begin{aligned}
r^{*}(x, t) & =\frac{x}{T+\tau_{0}-t}+f(t), \quad \text { if } x>0 \\
& =0, \quad \text { if } x=0
\end{aligned}
$$

We refer to this policy as Relaxed Optimal Policy (ROP).

The above solution has some interesting and intuitive features. First, the transmission rate at time $t$ for buffer size $x$ equals $x /\left(T+\tau_{0}-t\right)$, which is the least constant rate required to serve $x$ amounts of data by time $T+\tau_{0}$, plus an additional rate $f(t)$. This is natural as there is anticipation of future arrivals and the convexity of the cost function dictates that these (expected) future arrivals should be taken into account. Second, $f(t)$ depends on the underlying function $g($.$) (as$ observed by the dependence on $\alpha$ ). The intuition behind this is that if $g($.$) has a very fast increasing slope then its beneficial to$ reduce the buffer at a higher rate as data arriving close to the deadline will incur a lot of energy expenditure. Third, $r(x, t)$ depends only on the remaining time $T+\tau_{0}-t$. This follows from the fact that the Poisson process is memoryless and the future arrival statistics depends only on the remaining time.

Thus far, we have assumed $g(r)=\alpha^{r}-1, \alpha>1$. Proceeding as above we can also obtain solutions for other convex functions as well. One such example is $g(r)=r^{2}$ whose derivative is linear. For this function, the above methodology leads to a very intuitive solution and for which $f(t)$ is,

$$
f(t)=\frac{\lambda B(T-t)}{T+\tau_{0}-t}
$$

$\lambda B(T-t)$ is the expected future amount of data and $T+\tau_{0}-t$ is the time left. Thus the excess rate can be interpreted as the constant rate required to drain the future expected amount of data in the remaining time.

\section{Simulation Results}

We first compare the numerically obtained optimal solution and the ROP policy in (45). We, then, present simulation results comparing ROP with a non-anticipative policy, presented in Section III-C, that does not take into account the arrival statistics. Such a policy would simply drain the buffer at a rate $x(t) /\left(T-t+\tau_{0}\right)$. We call it the Simple Drain (SD) policy. This comparison shows the performance gains achieved by adjusting the rate in anticipation of future arrivals.

The optimality equations in (34)-(37) are solved numerically using a finite difference method. The partial differentials are approximated with a finite difference and the functions evaluated numerically starting from the boundaries. For a rigorous treatment of the convergence to the optimal solution see [14]. We know that $J(x, T)=\tau_{0} g\left(x / \tau_{0}\right), x \geq 0$. Approximating the partial differentials the optimality equations become,

$$
\begin{array}{r}
g^{\prime}\left(r^{*}(x, t)\right)=\frac{J(x, t+\delta t)-J(x-\delta x, t+\delta t)}{\delta x} \\
J(0, t)=g(0) \delta t+\lambda \delta t J(B, t+\delta t) \\
+(1-\lambda \delta t) J(0, t+\delta t)
\end{array}
$$
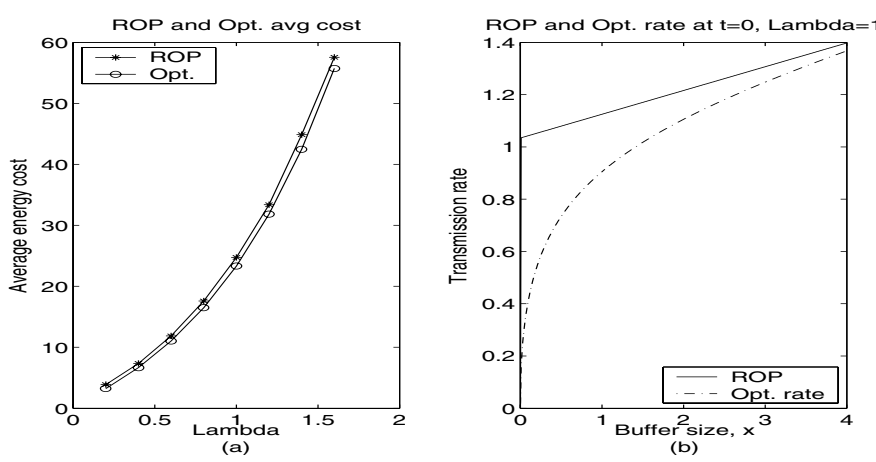

Fig. 7. (a) Comparison of expected energy cost for ROP and Optimal policy. (b) Comparison of rate at $t=0$ as a function of the buffer size $x$.

$$
\begin{array}{r}
\left(r^{*} / \delta x+1 / \delta t\right) J(x, t)=g\left(r^{*}\right)+\lambda\{J(x+B, t+\delta t) \\
-J(x, t+\delta t)\}+r^{*} \frac{J(x-\delta x, t)}{\delta x}+\frac{J(x, t+\delta t)}{\delta t}
\end{array}
$$

where $\delta x$ and $\delta t$ are the step sizes for $x$ and $t$ respectively. Starting at $t=T$ and iterating backwards, each time decrementing $t$ by $\delta t$, we can evaluate $J(0, t), r^{*}(x, t)$ and $J(x, t)$ for $x=(\delta x, . ., M \delta x)$. For the purposes of this paper we used the following parameters in the numerical evaluation; $T=10, \quad \tau_{0}=1, B=1, g(r)=\exp (r)-1, \delta x=$ $0.01, \delta t=0.02$. Figure 7(a) compares the optimal energy cost evaluated numerically with the expected energy cost for ROP at $t=0$ and $x_{0}=1$. The expected energy cost for ROP is obtained using simulations as explained later. As we see from the plot, ROP performs very close to the optimal. Figure 7(b) compares the optimal rate and the ROP rate (Eqn 45) as a function of the buffer size at $t=0$ and $\lambda=1$. As seen from the figure, at moderate buffer sizes the two rates are close and converge as $x$ increases but at very low buffer values the optimal rate is reduced as the boundary $x=0$ is closer and the boundary effect becomes prominent. The asymptotic (large $x$ ) convergence of the two rates is quite intuitive as the buffer non-negativity constraint becomes less important for large $x$.

We now present simulation results comparing ROP and SD policies with the following parameters. $T=10, \tau_{0}=1, B=$ $1, g(r)=\exp (r)-1$ and the initial buffer size $x_{0}=1$. The time interval $[0, T=10]$ is divided into discrete intervals of length $d t=10^{-3}$; thus, having 10,000 time slots. The arrival rate $\lambda$ is varied between $\lambda=0.2-1.6$ in steps of 0.2 . The Poisson arrival process is simulated using a Bernoulli model. In each time slot an arrival occurs with probability $\lambda d t$ and there are no arrivals with probability $1-\lambda d t$. At the beginning of each time slot the buffer size $x$ and the time $t$ is known. The rate of transmission in that slot for ROP is chosen from $(45)^{8}$ while the rate of transmission for SD as mentioned earlier is chosen as $\frac{x}{T-t+\tau_{0}}$. The same set of sample paths are applied for both the policies and the energy cost is computed as $\sum_{i} 10^{-3}\left(\exp \left(r_{i}\right)-1\right)+(\exp (x(T))-1)\left(\right.$ note $\left.\tau_{0}=1\right)$. The average is then taken over a set of $10^{4}$ sample paths.

\footnotetext{
${ }^{8}$ If $x$ is very small then the rate chosen might make the buffer go negative. In this case the rate is simply taken as $x / 10^{-3}$.
} 

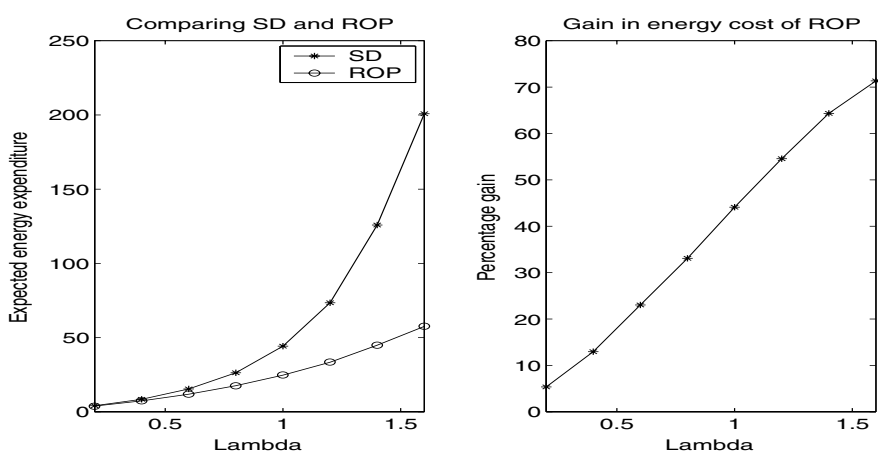

Fig. 8. Plot comparing the expected energy expenditure of ROP and SD and the percentage gain ((SDcost-ROPcost)*100/SDcost).
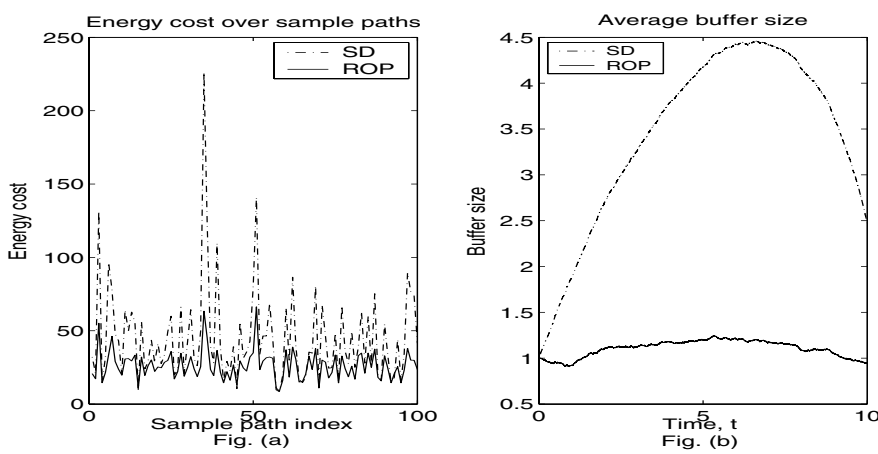

Fig. 9. (a) Comparison of energy expenditure for 100 sample paths at $\lambda=1$. (b) Comparison of average buffer size over time for $\lambda=1$.

Figure 8 compares the expected energy expenditure of the two policies for the set of $\lambda$ considered. As shown in the gain plot, ROP significantly outperforms SD policy and in fact the curve is upward sloping. Figure 9(a) plots the energy expenditure for the first 100 sample paths for $\lambda=1$. It is clear from the figure that ROP has lower energy cost than SD for almost all sample paths. Thus even on a sample path comparison ROP performs better. Finally, Figure 9(b) compares the average buffer size at time $t$ of the two policies for $\lambda=1$. As seen from the figure ROP tends to have a more uniform and much smaller average buffer size as compared to SD. Observe that for $\lambda=1$, on average a packet arrives in unit time and starting from $x_{0}=1$, ROP tends to transmit at that rate in an average sense. This conforms with the policy that would be optimal in case the arrivals were deterministic and uniform. As SD does not adjust rate in anticipation of arrivals, it transmits at low rates initially and hence the buffer tends to increase. Then, as the deadline gets closer the rate increases and the average buffer size drops.

\section{CONCLUSION}

We considered the problem of minimizing transmission energy over a finite time horizon with quality of service constraints. We formulated the problem using a calculus approach and posed it as a continuous time optimization. Our model encompasses both the packetized and continuous arrival flows. We obtained the optimal policy in the deterministic case and characterized its properties. We, then, considered a Poisson arrival process with a single deadline and modelled it as a stochastic optimal control problem. Based on the theory of stochastic differential equations and optimal control we obtained the optimality equations that can be solved numerically. Using a constraint relaxation we also obtained an elegant policy that outperformed a simple drain policy as shown by the simulations. Our novel formulation led us to obtain elegant solutions for an otherwise difficult problem. We believe that the approach holds great promise for future investigation. Incorporating other QoS constraints into the stochastic problem is actively pursued and extensions are investigated to other stochastic models as well.

\section{REFERENCES}

[1] Prabhakar B., Biyikoglu E.U., El Gamal A. "Energy-efficient transmission over a wireless link via lazy packet scheduling", INFOCOM 2001, vol. 1, pp 386 - 394, April 2001.

[2] Fu A., Modiano E., Tsitsiklis J. "Optimal energy allocation for delayconstrained data transmission over a time-varying channel", INFOCOM 2003, vol. 2, pp 1095 - 1105, April 2003.

[3] Khojastepour M., Sabharwal A. "Delay-constrained scheduling: power efficiency, filter design and bounds", INFOCOM 2004, March 2004.

[4] Nuggehalli P., Srinivasan V., Rao R.R. "Delay constrained energy efficient transmission strategies for wireless devices", INFOCOM 2002, vol. 3 , pp. 1765 - 1772,June 2002.

[5] Cruz R., "A Calculus for Network delay" (Parts I\&II),IEEE Transactions on Information Theory", Jan., 1991.

[6] Cruz R. "Quality of service guarantees in virtual circuit switched networks", IEEE JSAC, vol. 13, no.6, pp. 1048-1056, Aug. 1995.

[7] Berry R., Gallager R. "Communication over fading channels with delay constraints", IEEE Tran. on Information Theory, vol. 48, no. 5, May 2002.

[8] Collins B., Cruz R., "Transmission policies for time varying channels with average delay constraints", Proceedings, 1999 Allerton Conf. on Comm., Control and Comp., Monticello, IL, 1999.

[9] Ata B., "Dynamic power control in a wireless static channel subject to a quality of service constraint", To appear in Operations Research.

[10] Gradshteyn I.S., Ryzhik I.M., "Table of Integrals, Series and Products", Academic Press, San diego, 2000.

[11] Bertsekas D., Gallager R., Data Networks, $2^{\text {nd }}$ Edn., Prentice Hall.

[12] Kushner H., Stochastic Stability and Optimal Control, Academic Press, NY, 1967.

[13] Davis M., Markov Models and Optimization, Chapman and Hall, 1993.

[14] Kushner H., Dupuis P., Numerical Methods for Stochastic Control Problems in Continuous Time, Springer-Verlag, 1992.

\section{APPENDIX I}

\section{PROOF OF OPTIMALITY OF THE ALGORITHM}

From Theorem II we know that $D^{o p t}(t)$ is unique. Hence it suffices to prove that (a) at every iteration one of the steps of the algorithm is satisfied and (b) the constructed curve satisfies the optimality criterion.

Proof of claim $(a)$ : At every feasible point $\left(t_{0}, \alpha\right), \beta_{0}$ is defined as in Section III-C. Line $L_{0}$ is either tangent to $A(t)\left(\right.$ or $\left.D_{\min }(t)\right)$ or not. If it is not tangent then by Lemma 3 it must intersect $A(t)$ or $D_{\min }(t)$ first and step (2) of the algorithm is then satisfied. If $L_{0}$ is tangent then step (3) is followed. Finally the new point $\left(t_{1}, \gamma\right)$ obtained from the algorithm is also feasible.

Proof of claim $(b)$ : Let $D_{c}(t)$ denote the constructed curve. Its obvious from the construction that at all points where the slope changes Corollary I. 1 is satisfied. We next show that Corollaries I.2-I.3 are also satisfied. Let $t_{0}$ be the starting 
instant at some iteration. Let step 2 be satisfied at $t_{0}$ then the sets $\mathcal{F}_{D_{m}}$ and $\mathcal{F}_{A}$ are non-empty and $L_{o}$ lies in $\mathcal{F}$. Suppose $L_{o}$ intersects $D_{\min }(t)$ first, i.e. at $t_{1}$ (as in the algorithm) we have $L_{o}\left(t_{1}\right)=D_{\min }\left(t_{1}\right)$ (and $L_{o}\left(t_{1}\right) \neq A\left(t_{1}^{-}\right)$). Since $L_{o}$ does not intersect $A(t)$ in $\left[t_{0}, t_{1}\right]$, if we pick a $L_{1} \in F_{A}$ with slope close to $L_{o}^{\prime}\left(=\beta_{o}\right)$ then $L_{1}$ would intersect $A(t)$ beyond $t_{1}$. More precisely, there exist lines $L_{1} \in F_{A}$ with slope $\beta_{o}<L_{1}^{\prime}<\beta_{o}+\epsilon$ for some $\epsilon>0$ such that $L_{1}$ intersects $A(t)$ first at $\tilde{t}>t_{1}$. It then follows that at the next iteration starting at $t_{1}$ the optimal line cannot have slope greater than $\beta_{o}$ and Corollary I.2 is satisfied at $t_{1}$. Similarly, if at $t_{1}$ we have $L_{o}\left(t_{1}\right)=A\left(t_{1}\right)\left(\right.$ or $\left.A\left(t_{1}^{-}\right)\right)$then we can show that Corollary I.3 is satisfied at $t_{1}$. Note that if at $t_{1}$ we have $L_{o}\left(t_{1}\right)=D_{\min }\left(t_{1}\right)=A\left(t_{1}^{-}\right)$then it does not matter how the slope changes beyond $t_{1}$. Now, instead let step 3 be satisfied at $t_{0}$ then $L_{o}$ is tangent to $D_{\min }(t)($ or $A(t))$ and by an analogous argument as above we can show that the result holds for all $t \in\left(t_{0}, t_{1}\right]$. Finally, as $t_{0}$ is arbitrary, starting at $(0,0)$, it follows that Corollaries I.1-I.3 are all satisfied. This implies that in a small interval around every point where the slope of $D_{c}(t)$ changes we cannot construct a feasible line segment and hence $D_{c}(t)$ satisfies the optimality criterion.

\section{APPENDIX II \\ PROOF OF THEOREM III}

Consider a feasible departure curve $D(t) \in \Gamma$ that is not optimal. Let $[a, b]$ be the interval over which the optimality criterion is violated. Then, based on the construction in Theorem I we obtain a new curve $\tilde{D}(t)$ that continues to satisfy the non-decreasing, continuity and feasibility properties. The line segment $L(t)$ between $[a, b]$ in $\tilde{D}(t)$ always has a slope that is less than the maximum slope of $D(t)$ between $[a, b)$. As $\tilde{D}(t)=D(t), t \notin(a, b)$, the overall maximum slope of $\tilde{D}(t)$ cannot exceed that of $D(t)$.

$$
\max _{t \in[0, T)} \tilde{D}^{\prime}(t) \leq \max _{t \in[0, T)} D^{\prime}(t)
$$

If $\tilde{D}(t)=D^{o p t}(t)$ then we are done. If not then repeat the process for $\tilde{D}(t)$ now. For $g($.$) strictly convex, the energy$ expenditure strictly decreases at each iteration. Thus, we obtain a sequence of curves with decreasing energy metric that is lower bounded by the optimal cost. As the optimal curve that achieves the lower bound is unique, it follows that the above sequence eventually converges to $D^{\text {opt }}(t)$. The result then follows from a repeated application of (50).

\section{APPENDIX III}

\section{BOUNDARY CONDITION}

To satisfy the non-negativity constraints the rate, $r(x, t)$, must be zero when $x=0$. Start at some point $(x=0, t<$ $T$ ) on the boundary. Let $\gamma>t$ be the first packet arrival instant after $t$. Let $\tau=\gamma \wedge T$, where $\wedge$ denotes the minimum operation. Consider $\delta>0$ and let $\tilde{t}=t+\delta$, then, from the Markov property of the process we can write $J(0, t)$ as,

$$
J(0, t)=E\left[\int_{t}^{\tilde{t} \wedge \tau} g(0) d s+J\left(x_{\tilde{t} \wedge \tau}, \tilde{t} \wedge \tau\right)\right]
$$

Using the indicator functions $I_{(\tau<\tilde{t})}$ and $I_{(\tau>\tilde{t})}=1-I_{(\tau<\tilde{t})}$ to condition on the respective events we can re-write (51) as,

$$
g(0)+\frac{E\left[J\left(x_{\tilde{t}}, \tilde{t}\right)\right]-J(0, t)}{\delta}+\frac{1}{\delta} E\left[h(\tilde{t}, \tau) I_{(\tau \leq \tilde{t})}\right]=0
$$

where $h(\tilde{t}, \tau)=J\left(x_{\tau}, \tau\right)-J\left(x_{\tilde{t}}, \tilde{t}\right)-\int_{\tau}^{\tilde{t}} g(0) d s$. Consider the event $\tau \leq \tilde{t}$, then, as $\delta \downarrow 0, E h(\tilde{t}, \tau) \rightarrow 0$ and $\frac{P(t<\tau \leq \tilde{t})}{\delta} \rightarrow \lambda$. Hence, the third term in the above expression goes to zero. Also in the limit $\delta \downarrow 0$, the second term is the differential generator as in (27) with $x=0$ and $r=0$. Thus we get,

$$
\begin{gathered}
g(0)+\frac{\partial J(0, t)}{\partial t}+\lambda\{J(B, t)-J(0, t)\}=0, t \in[0, T) \\
\text { APPENDIX IV } \\
\text { ProOFS OF LEMMAS }
\end{gathered}
$$

Proof of Lemma 2: Take two lines $L_{1}(t), L_{2}(t) \in \mathcal{F}$ with slopes $s_{1}, s_{2}$ respectively. Without loss of generality, let $s_{1}>$ $s_{2}$. Let $\epsilon_{1}$ and $\epsilon_{2}$ be the respective durations over which they are feasible. Take $\epsilon=\min \left[\epsilon_{1}, \epsilon_{2}\right]$, then, over $\left[t_{0}, t_{0}+\epsilon\right)$ we can view $L_{1}(t), L_{2}(t)$ equivalently as new $A(t)$ and $D_{\min }(t)$ respectively. Any line with slope $s$ such that $s_{2} \leq s \leq s_{1}$ is then feasible for duration $\epsilon$ and hence belongs to $\mathcal{F}$.

Proof of Lemma 4: (a) Let $\tilde{t}$ be the point at which $L_{D}(t)$ intersects $D_{\min }(t)$ first. By definition, $L_{D}(t)<A(t), \forall t \in$ $\left(t_{0}, \tilde{t}\right)$. The proof now follows in two parts. First, we show that any line in $\mathcal{F}$ with slope less than $L_{D}^{\prime}$ must intersect $D_{\min }(t)$ before $\tilde{t}$ and second that this line does not intersect $A(t)$ in $\left(t_{0}, \tilde{t}\right)$. Consider $L(t) \in \mathcal{F}$ with slope less than $L_{D}^{\prime}$ then $L(t)<L_{D}(t), \forall t>t_{0}$. Hence, at time $\tilde{t}$ we have $L(\tilde{t})<L_{D}(\tilde{t})=D_{\min }(\tilde{t})^{9}$ and this implies that $L(t)$ becomes infeasible and below $D_{\min }(t)$ at $\tilde{t}$. Therefore $L(t)$ must intersect $D_{\min }(t)$ before $\tilde{t}$. Next, since $L(t)<L_{D}(t)<A(t)$ in $t \in\left(t_{0}, \tilde{t}\right)$, the line $L(t)$ cannot intersect $A(t)$ first. Along similar lines we can prove (b). Its proof is omitted for brevity.

Proof of Lemma 5: We can re-write (43) as,

$$
\frac{d}{d t}\left(\left(T-t+\tau_{0}\right) f(t)\right)=-\frac{\lambda\left(T-t+\tau_{0}\right)}{\ln (\alpha)}\left(\alpha^{\frac{B}{T+\tau_{0}-t}}-1\right)
$$

Taking a Taylor series expansion of $\alpha^{\frac{B}{T+\tau_{0}-t}}-1$ and using Monotone Convergence Theorem we get,

$$
\left(T-t+\tau_{0}\right) f(t)=\sum_{n=1}^{\infty} \int-\frac{(\lambda / \ln (\alpha))(B \ln (\alpha))^{n}}{n !\left(T-t+\tau_{0}\right)^{n-1}} d t+c
$$

where $c$ is the constant of integration. Integrating each term and substituting $f(T)=0$ we get the result. The series term in (44) which is denoted as $S_{m}$ has non-negative terms and hence is non-decreasing. For $t \leq T, S_{m} \leq \sum_{n=3}^{m} \frac{(B \ln (\alpha))^{n} \tau_{0}^{2}}{n ! \tau_{0}^{n}} \leq$ $\sum_{n=0}^{m} \frac{(B \ln (\alpha))^{n} \tau_{0}^{2}}{n ! \tau_{0}^{n}} \stackrel{m \rightarrow \infty}{\longrightarrow} \tau_{0}^{2} \exp \left(B \ln (\alpha) / \tau_{0}\right)$. Thus the series is convergent.

\footnotetext{
${ }^{9}$ If $\tilde{t}$ is the discontinuity point then $L_{D}(t)-D_{\min }(t)$ changes sign at $\tilde{t}$ and so $L(t)-D_{\min }(t)$ must have changed sign earlier at $t \leq \tilde{t}$.
} 